\title{
An empirical constitutive law for concentrated colloidal suspensions in the approach of the glass transition
}

\author{
H. Henning Winter • Miriam Siebenbürger • \\ David Hajnal • Oliver Henrich • \\ Matthias Fuchs - Matthias Ballauff
}

Received: 13 January 2009 / Accepted: 3 July 2009 / Published online: 29 July 2009

\begin{abstract}
Concentrated, non-crystallizing colloidal suspensions in their approach of the glass state exhibit distinct dynamics patterns. These patterns suggest a powerlaw rheological constitutive model for nearglass viscoelasticity, as presented here. The rheological parameters used for this model originate in the mode-coupling theory. The proposed constitutive model provides explicit expressions for the steady shear viscosity, the steady normal stress coefficient, the modulus-compliance relation, and the $\alpha$ peak of $G^{\prime \prime}$. The relaxation pattern distinctly differs from gelation.
\end{abstract}

Keywords Glass transition - Mode-coupling theory • Colloidal glass - BSW spectrum - Linear viscoelasticity $\cdot$ Near-glass dynamics

\section{Introduction}

Recent experimental and theoretical advances allow a new look at the rheology of the glass transition, a

H. H. Winter (ख)

Chemical Engineering, University of Massachusetts,

Amherst, MA 01003, USA

e-mail: winter@ecs.umass.edu

M. Siebenbürger $\cdot$ M. Ballauff

Physikalische Chemie I, Universität Bayreuth, 95440 Bayreuth, Germany

D. Hajnal · O. Henrich · M. Fuchs

Fachbereich Physik, Universität Konstanz, 78457 Konstanz, Germany fundamental phenomenon of nature, which leads to the slow-down of the internal dynamics in a material and to the eventual arrest of internal motion of the material's constituents. This study focuses on the rheological patterns of glass-forming fluids in the approach of the glass transition (denoted as "near-glass" dynamics in the following), as observed by Siebenbürger et al. (2009), who used colloidal suspensions as model systems for molecular glasses to measure the characteristic changes of the rheological properties across the fluid-to-glass transition. Their findings justify a further analysis since the experiments compare well with mode-coupling theory (MCT) predictions.

A deeper understanding of many aspects of the glass transition has been accomplished by MCT (Götze and Sjögren 1992). Recently, MCT has been shown to account for the non-linear rheology of concentrated colloidal suspensions (Fuchs and Cates 2002). In particular, MCT explains the linear viscoelasticity in the approach of the glass from the fluid state. At higher densities, experiments by Crassous et al. (2008a) and Brambilla et al. (2009) have shown that the ideal glass cannot be reached because of an unexplained long-time process, which cuts off the glassy nonergodicity very close to $\varepsilon=0$. MCT neglects this ergodicity restoring process (see, however, Schweizer 2007, Schweizer and Saltzman 2003, and Mayer et al. 2006 for recent extensions). The proposed constitutive model of this study concerns the dynamics at concentrations that are not yet affected by this long-time process.

The distance from the glass as defined by the separation parameter, $\varepsilon=\left(\phi-\phi_{\mathrm{c}}\right) / \phi_{\mathrm{c}}$, serves as an independent parameter of near-glass dynamics. The critical volume fraction of particles, $\phi_{c}$, depends on particlesize distribution. For monodisperse systems, it assumes 
the value $\phi_{\mathrm{c}}=0.58$. The glass is defined by $\varepsilon=0$. Nearglass states (fluids) have negative $\varepsilon$, and soft glassy states (solids) have positive $\varepsilon$. This study focuses on fluid states and is limited to $\varepsilon<0$ (near-glass).

The general problem of solidification of complex materials and its rheological consequences has been studied extensively (Ferry 1980; Larson 1999). Examples are gelation with the critical relaxation modulus $G(t)=S t^{-n_{\mathrm{cg}}}$ at gel point (Winter and Mours 1997) and crystallization. Needed is a complete rheological description of the fluid-to-glass transition that is based on the knowledge of the relaxation time spectrum $H(\tau)$ or the relaxation modulus $G(t)$ in Boltzmann's linear viscoelastic constitutive equation for the stress:

$$
\begin{aligned}
& \sigma(t)=\int_{-\infty}^{t} d t^{\prime} G\left(t-t^{\prime}\right) \dot{\gamma}\left(t^{\prime}\right) \text { with } \\
& G(t)=G_{\mathrm{e}}+\int_{0}^{\tau_{\max }} \frac{d \tau}{\tau} H(\tau) e^{-t / \tau}
\end{aligned}
$$

The upper limit of the relaxation time spectrum, $\tau_{\max }$, belongs to the largest connected region in the material that can still relax. This study focuses on the fluid behavior for which the equilibrium modulus is zero, $G_{\mathrm{e}}=$ 0 . Either one of the material properties, $H(\tau)$ or $G(t)$, is sufficient to fully describe the linear viscoelasticity of a material in general (Boltzmann 1874). $H(\tau)$ or $G(t)$ are typically measured indirectly by performing smallamplitude oscillatory shear experiments to obtain the dynamic moduli

$$
\begin{aligned}
G^{\prime}(\omega) & =G_{e}+\int_{o}^{\tau_{\max }} \frac{d \tau}{\tau} H(\tau) \frac{(\omega \tau)^{2}}{1+(\omega \tau)^{2}} ; \\
G^{\prime \prime}(\omega) & =\int_{0}^{\tau_{\max }} \frac{d \tau}{\tau} H(\tau) \frac{(\omega \tau)}{1+(\omega \tau)^{2}} .
\end{aligned}
$$

and backing out $H(\tau)$ or $G(t)$ from these (Baumgärtel and Winter 1989). This is the approach taken here. Equation 2 is obtained by inserting the periodic shear rate into Eq. 1 (Ferry 1980).

Here, we demonstrate that $H(\tau)$ can be obtained from experimental data and from MCT for near-glass material states. We begin by proposing a powerlaw constitutive equation and by applying it to describe dynamic mechanical experiments of a concentrated colloidal suspension. Rheological parameters will be shown to originate from MCT. Based on Boltzmann's equation of linear viscoelasticity, rheological properties will be predicted such as zero shear viscosity, normal stress function, creep compliance, alpha relaxation, and $G^{\prime \prime}$ minimum and maximum.

\section{Linear viscoelasticity of near-glassy states}

Two pronounced relaxation processes, known as $\alpha$ and $\beta$-relaxation, each with its distinct pattern of relaxation times and strengths, govern the near-glass dynamics. As most pronounced phenomenon, the dynamics slows down close to the critical volume fraction $\phi_{\mathrm{c}}$. MCT successfully predicts near-glass properties for concentrated, non-crystallizing colloidal suspensions (Bengtzelius et al. 1984; Götze 1991; Götze and Sjögren 1992). The fast $\beta$ relaxation originates from the localized motion of particles inside cages that are formed by their neighboring particles. The slow $\alpha$ relaxation expresses the breakup of particle cages. MCT provides a self-consistent formulation for the time-autocorrelation function of density fluctuations, which is of general interest as it is directly accessible in experiments and allows detailed insight into the collective dynamics of dense fluids.

van Megen et al. (1991) and van Megen and Underwood (1994) performed extensive experiments with hard-sphere suspensions to check and verify the predictions of MCT. The cornerstone of MCT is a nonlinear feedback mechanism, which captures the cage effect among the constituting particles in dense fluids. In a fluid near the glass transition, particles can move freely and correlation functions decay quickly to zero. However, on increasing the density $\phi$, or alternatively in thermal systems on lowering the temperature (molecular glass former), the motion of a particle gets more and more hindered by its neighboring particles, which behave as cage-forming obstacles. The dynamics undergoes a qualitative change from rather independent to a collective one, in which an individual particle can only move when its neighbors move accordingly and open up free space. Particle movements in these systems become more sluggish, and the characteristic time scales for relaxation processes begin to grow significantly. Then, below a critical temperature or above a critical density, the system undergoes a transition to a state, in which correlations do not decay to zero anymore. The particles have been trapped inside the cages formed by their neighbors, and they can only move if the whole cage moves with them. As each cage-forming particle is, in turn, caged inside another cage, a nearly complete dynamical arrest sets in when arriving at the glass.

Suspensions of hard spheres or of particles characterized by a sufficiently steep mutual repulsion may be treated as the most simple model system for 


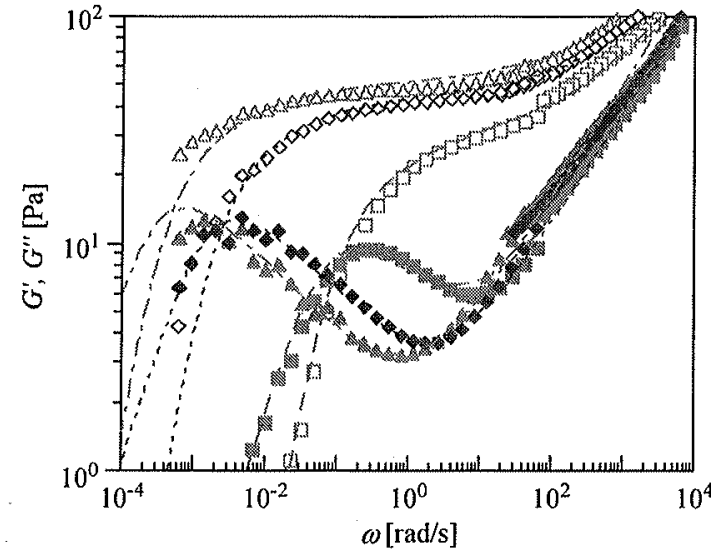

Fig. $1 G^{\prime} G^{\prime \prime}$ data of Siebenbürger et al. (2009) for three volume fractions. Data as discrete points in the figure on the left side are expressed with the spectrum of Eq. 5 below (lines in the figure on the left side). The corresponding three continuous spectra according to Eq. 5 are shown in the figure on the right side. The

studying near-glass dynamics (van Megen et al. 1991; Mason and Weitz 1995). Most advantageous are model suspensions with particles of tunable size. Crassous et al. (2006a, b, 2008b) and Siebenbürger et al. (2009) achieved such tunability by coating spherical polystyrene particles (diameter $\sim 100 \mathrm{~nm}$ ) with a crosslinked shell of thermosensitive $\operatorname{poly}(N$ isopropylacrylamide) (PNIPAM). Dense suspensions can be generated in situ since the PNIPAM shell of these particles swells when immersed in cold water $\left(10-15^{\circ} \mathrm{C}\right)$ and, vice versa, water gets expelled at higher temperatures, leading to a considerable shrinking. Due to a slight polydispersity of the particles, crystallization is suppressed and no fluid-crystal transition intervenes. However, the size distribution is still narrow enough to achieve a distinct separation of the $\alpha$ and $\beta$ relaxations. In the concentration range of this study, all previous history of the sample can be erased (without mechanical deformation) by temporarily raising the temperature and, thus, lowering the volume fraction to the fluid

Table 1 BSW parameters for data of Siebenbürger et al. (2009)

\begin{tabular}{llllll}
\hline$\varepsilon$ & -0.07200 & -0.00350 & -0.00042 & -0.00017 & 0.000021 \\
$G_{\mathrm{c}}[\mathrm{Pa}]$ & $1.62 \mathrm{E}+01$ & $3.02 \mathrm{E}+01$ & $4.17 \mathrm{E}+01$ & $4.68 \mathrm{E}+01$ & $5.25 \mathrm{E}+01$ \\
$\tau_{\alpha}[\mathrm{s}]$ & $2.04 \mathrm{E}-02$ & $1.38 \mathrm{E}+01$ & $6.60 \mathrm{E}+02$ & $3.39 \mathrm{E}+03$ & $1.91 \mathrm{E}+04$ \\
$\tau_{0}[\mathrm{~s}]$ & $2.57 \mathrm{E}-03$ & $1.66 \mathrm{E}-03$ & $1.29 \mathrm{E}-03$ & $1.82 \mathrm{E}-03$ & $4.07 \mathrm{E}-03$ \\
$n_{\alpha}$ & 0.36 & 0.36 & 0.36 & 0.36 & 0.36 \\
$n_{\beta}$ & 0.5 & 0.5 & 0.5 & 0.5 & 0.5 \\
\hline
\end{tabular}

For the sake of clarity, only the middle three data sets are used in Fig. 1

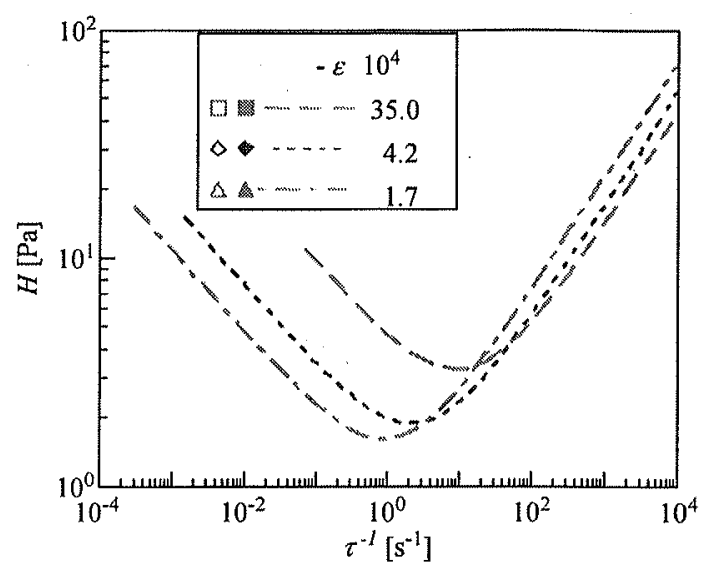

fit is achieved by adjusting the BSW parameters with the IRIS graphics tool (Winter and Mours 2006). A good fit requires that $n_{\alpha}<n_{\beta}$. The powerlaw spectrum is less successful for the highconcentration rheology data

regime. Siebenbürger et al. (2009) presented dynamic mechanical data of such suspension of thermosensitive particles, see Fig. 1. As will be shown below, a powerlaw spectrum (BSW spectrum) is able to express the data. The spectrum mimics the loss modulus over a wide frequency range. The exponent values determine the slopes and can be read directly from the $G^{\prime \prime}(\omega)$ plot. The fit parameters are listed in Table 1.

\section{Empirical constitutive equation for the near-glass}

The $\alpha$ relaxation is known to express itself in a maximum of the loss modulus, $G_{\alpha, \text { max }}^{\prime \prime}$. MCT predicts that the peak value is independent of $\varepsilon$ while the peak position shifts to lower and lower frequencies in the approach of the glass (Fig. 2). Powerlaw asymptotes, $G^{\prime \prime} \sim \omega \tau_{\alpha}$ and $G^{\prime \prime} \sim\left(\omega \tau_{\alpha}\right)^{-n_{\alpha}}$, appear on the low-frequency and the high-frequency sides of the $G^{\prime \prime}$ peak, respectively. The narrowness of the peak and the powerlaw asymptotes on either side suggest a powerlaw relaxation time spectrum for the long time behavior ( $\alpha$ relaxation)

$H_{\alpha}=n_{\alpha} G_{c}\left(\frac{\tau}{\tau_{\alpha}(\varepsilon)}\right)^{n_{\alpha}}$ for $\tau<\tau_{\alpha}(\varepsilon)$ and $\varepsilon<0$

The spectrum is assumed to be cut off at its longest relaxation time $\tau_{\alpha}$, i.e., the spectrum vanishes beyond $\tau_{\alpha}\left(H_{\alpha}=0\right.$ for $\left.\tau>\tau_{\alpha}\right)$.

Because of the dominance of the $\alpha$ relaxation, much less experimental information is available for the short 


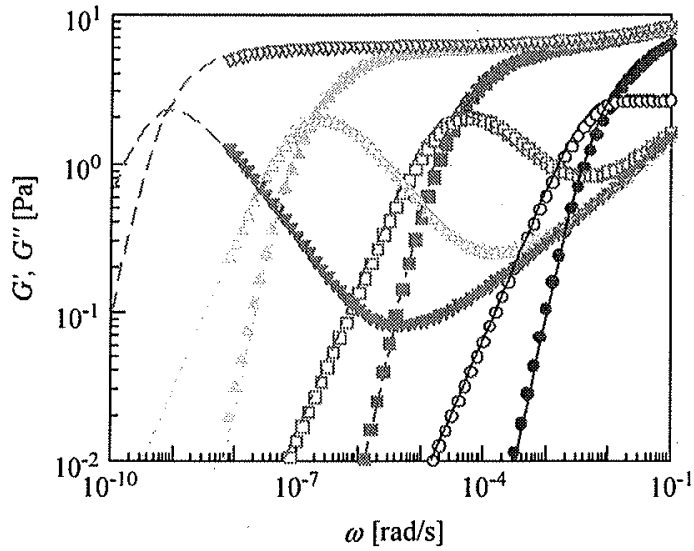

Fig. 2 On the left side: MTC predictions of $G^{\prime}, G^{\prime \prime}$ near the glass (data points) and corresponding fit (lines through data points) with BSW relaxation time spectra. All MCT curves were calculated using the schematic $F_{12}^{\left.()^{\prime}\right)}$ model of Siebenbürger et al. (2009) and setting $v_{\sigma}=70, \Gamma=80, \gamma_{c}=0.1414, \eta_{\infty}^{\omega}=0.3938$ and $\eta_{\infty}^{y}=0.8313$. As the only parameter, the separation parameter

time behavior ( $\beta$ relaxation). Near the glass transition, it might be approximated as

$H_{\beta}=n_{\alpha} G_{\mathfrak{c}}\left(\frac{\tau}{\tau_{0}}\right)^{-n_{\beta}}$

because it should follow the powerlaw that can be expected right at the transition (at $\varepsilon=0$ ), when the particles explore the cages that are about to close. In the literature, $\tau_{0}$ is called the "matching time." The easiest ansatz for combining $\alpha$ and $\beta$ relaxation is by linear superposition: this leads to the proposed rheological constitutive model of near-glass dynamics (for $\varepsilon<0$ )

$$
H(\tau, \varepsilon)=n_{\alpha} G_{\mathfrak{c}}\left\{\left(\frac{\tau}{\tau_{\alpha}(\varepsilon)}\right)^{n_{\alpha}}+\left(\frac{\tau}{\tau_{0}}\right)^{-n_{\beta}}\right\}
$$$$
\text { for } \tau<\tau_{\alpha}(\varepsilon) \text { and } \varepsilon \leq 0 \text {. }
$$

As is shown in Fig. 1, this spectrum is able to describe the linear viscoelastic data in the approach of the glass. The validity and properties of the above spectrum will be explored in the remainder of the paper.

The spectrum of Eq. 5 is otherwise known as BSW spectrum (Baumgärtel et al. 1990), and its properties have been mapped extensively (Carri and Winter 1997; Friedrich et al. 2008). The BSW spectrum is remarkable in its simplicity since it does not introduce any new parameter beyond the characteristic parameters of the $G^{\prime}, G^{\prime \prime}(\omega)$ pattern. The crossover between $\alpha$ and $\beta$ regions results in a minimum of $G^{\prime \prime}$. This minimum shifts

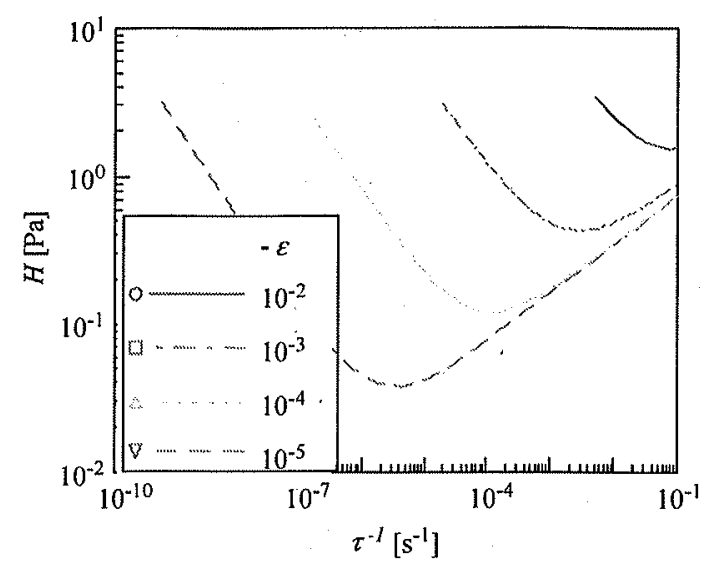

$\varepsilon$ was varied from $-10^{-2}$ to $-10^{-5} . H(\tau)$ is shown on the right side. The nearly complete agreement over several decades of frequency suggests that the powerlaw format of the relaxation spectrum is appropriate. The powerlaw spectrum parameters for these plots are all fixed: $n_{\alpha}=0.63 ; G_{\mathrm{c}}=5 \mathrm{~Pa} ; \tau_{\alpha} \sim(-\varepsilon)^{-2.4}$; $\tau_{0}=0.33 \mathrm{~s}$

to lower frequencies when the distance to the glass gets small ( $\varepsilon$-dependent crossover), see further below.

\section{MCT prediction of powerlaw relaxation parameters}

The single set of well-defined parameters of MCT can provide a full description of the viscoelastic behavior of near-glass suspensions. This will be described next by already using the notation of the rheological constitutive equation, Eq. 5. The $\alpha$ - and $\beta$-relaxation processes of near-glass dynamics (with $\varepsilon<0$ ) are both dominated by the plateau modulus

$G_{\mathrm{c}}=g_{0} \frac{k_{\mathrm{B}} T}{R_{\mathrm{H}}^{3}}$.

The recent experiments of Crassous et al. (2008a) confirmed this relation in the approach of $\phi \rightarrow \phi_{c}$ from below. MCT predicts $G_{\mathrm{c}}$ as a function of the particle size $\left(R_{\mathrm{H}}\right)$ and thermal energy, while the prefactor $g_{0}$ is a pure number that can be calculated from the frozenin structure when reaching the glass state $(\varepsilon=0)$; it is quantified by the glass form factor (non-ergodicity parameter) $f_{\mathrm{q}}$ (Götze 1991). Light scattering measurements of $f_{\mathrm{q}}$ confirmed the MCT prediction (van Megen et al. 1991), and flow curve measurements verified the magnitude of $G_{\mathrm{c}}$ for (almost) monodisperse hard spheres (Crassous et al. 2008a). 
The characteristic relaxation times for the $\alpha$ relaxation are predicted to diverge in a powerlaw in the approach of the glass (for $\varepsilon \leq 0$ ) (Götze 1991; Götze and Sjögren 1992)

$\tau_{\alpha}=\tau_{0, \alpha}(-\varepsilon)^{-\gamma}$

The characteristic relaxation time for the $\beta$ relaxation is constant. A prediction of the two time constants ( $\tau_{0}$ and $\tau_{0, \alpha}$ ) requires the full $\mathrm{MCT}$ calculation.

MCT predicts a coupling between $\alpha$ and $\beta$ relaxation through the two-relaxation exponents $n_{\alpha}$ and $n_{\beta}$ as (Götze 1991; Götze and Sjögren 1992)

$\frac{\Gamma^{2}\left(1+n_{\alpha}\right)}{\Gamma\left(1+2 n_{\alpha}\right)}=\lambda=\frac{\Gamma^{2}\left(1-n_{\beta}\right)}{\Gamma\left(1-2 n_{\beta}\right)}$,

which also determines the exponent, $\gamma$, of the $\alpha$ relaxation times (Eq. 7)

$\gamma=\frac{1}{2}\left(\frac{1}{n_{\alpha}}+\frac{1}{n_{\beta}}\right)$

$\Gamma$ is the familiar gamma function. If one of the three exponents is given, the other two exponents follow according to the MCT. $n_{\alpha}$ is known as the von Schweidler exponent (von Schweidler 1907; Götze 1991; Götze and Sjögren 1992) and $n_{\beta}$ as the critical powerlaw exponent; MCT finds $n_{\alpha}>n_{\beta}$.

The set of BSW parameters, Eq. 5, are completely defined by the MCT. The cut-off or longest relaxation time $\tau_{\max }=c_{\mathrm{G}} \tau_{\alpha}(\varepsilon)$ may differ by a factor of order unity from $\tau_{\alpha}(\varepsilon)$ itself as defined for MCT density correlations by Franosch and Götze (1999). The calculation of $c_{\mathrm{G}}$ requires the full MCT equations (Fuchs et al. 1992), as $c_{G}$ and the detailed shape of the spectrum around $\tau_{\alpha}(\varepsilon)$ depend on the quantity (wave vector) under consideration. We set $c_{\mathrm{G}}=1$ for simplicity, exactly recovering the BSW spectrum of Eq. 5 .

\section{Properties of the $\alpha$ relaxation of near-glass dynamics}

The powerlaw spectrum of Eq. 3 ( $\alpha$ relaxation) dominates the dynamics in the approach of the glass, and the material exhibits the following linear viscoelastic properties:

Steady shear viscosity $\eta_{\mathrm{o}}=\frac{n_{\alpha}}{1+n_{\alpha}} G_{\mathrm{c}} \tau_{\alpha} \sim(-\varepsilon)^{-\gamma}$,
Steady shear first normal stress coefficient

$$
\Psi_{1,0}=\frac{2 n_{\alpha}}{2+n_{\alpha}} G_{\mathrm{c}} \tau_{\alpha}^{2} \sim(-\varepsilon)^{-2 \gamma},
$$

Characteristic time constant

$$
\tau_{\mathrm{c}}=\frac{\Psi_{1,0}}{2 \eta_{\mathrm{o}}}=\frac{1+n_{\alpha}}{2+n_{\alpha}} \tau_{\alpha \alpha} \sim(-\varepsilon)^{-\gamma},
$$

Creep compliance $J(t)=\frac{1}{G(t)} \frac{\left(1+n_{\alpha}\right)^{2}}{n_{\alpha}\left(2+n_{\alpha}\right)}$,

$\alpha$-relaxation peak (Friedrich et al. 2008):

$$
G^{\prime \prime} \text { maximum } G_{\alpha, \max }^{\prime \prime} \approx \frac{G_{\mathrm{c}} n_{\alpha}}{1+n_{\alpha}^{2}}
$$

location of $G^{\prime \prime}$ maximum

$$
\omega_{\alpha, \max } \cong \frac{1}{\tau_{\alpha}} \frac{1}{n_{\alpha}} \sqrt{\frac{2 n_{\alpha}+1}{n_{\alpha}+1}} \approx \frac{1}{\tau_{\alpha} n_{\alpha}} \approx(-\varepsilon)^{\gamma} .
$$

The dynamic moduli of the $\alpha$ relaxation, $G_{\alpha}^{\prime}$ and $G_{\alpha}^{\prime \prime}$, approach asymptotes at the low frequencies

$$
\begin{aligned}
& G_{\omega \tau_{\alpha<<<}}^{\prime}(\omega)=\frac{n_{\alpha}}{2+n_{\alpha}} G_{\mathrm{c}}\left(\omega \tau_{\alpha}\right)^{2} \\
& G_{\omega \tau_{\alpha<<<1}^{\prime \prime}}^{\prime \prime}(\omega)=\frac{n_{\alpha x}}{1+n_{\alpha}} G_{\mathrm{c}} \omega \tau_{\alpha \chi} .
\end{aligned}
$$

The corresponding high-frequency asymptotes are ( $\alpha$ relaxation only)

$$
\begin{aligned}
& G_{\omega \tau_{\alpha>>1}}^{\prime}(\omega)=G_{\mathbf{c}} \\
& G_{\omega \tau_{\alpha>>1}}^{\prime \prime}(\omega)=\frac{\pi / 2}{\cos \left(n_{\alpha} \pi / 2\right)} n_{\alpha} G_{\mathfrak{c}}\left(\omega \tau_{\alpha}\right)^{-n_{\alpha}}
\end{aligned}
$$

\section{Properties of the $G^{\prime \prime}$ minimum of near-glass dynamics}

The pronounced minimum of the crossover of $\alpha$ and $\beta$ relaxations occurs at a characteristic $\beta$-relaxation time that Franosch and Götze (1999) had defined for density correlations. Appealing to experiments is the accessibility of the minimum; see also Koumakis et al. (2008). This $\beta$-relaxation time is now calculated as

$\tau_{\beta}=\tau_{0}(-\varepsilon)^{-1 / 2 n_{\beta}}$. 
It appears in Eq. 5 when rewritten as a homogeneous function

$$
\begin{aligned}
& H^{\mathrm{MCT}}(\tau, \varepsilon)=n_{\alpha} G_{\mathrm{c}} \sqrt{-\varepsilon}\left\{B\left(\frac{\tau}{\tau_{\beta}(\varepsilon)}\right)^{n_{\alpha}}+\left(\frac{\tau}{\tau_{\beta}(\varepsilon)}\right)^{-n_{\beta}}\right\} \\
& \text { for } \tau<\tau_{\alpha}(\varepsilon) \text { and } \varepsilon<0,
\end{aligned}
$$

with parameter $B$ of order unity and connected to $\tau_{\alpha, 0}$ and $\tau_{0}$. The time scale $\tau_{\beta}(\varepsilon)$ can be read off from the minimum in $G^{\prime \prime}(\omega)$, which shifts to lower frequencies with $1 / \tau_{\beta}$ and becomes deeper with $\sqrt{-\varepsilon}$ for approaching the glass transition $\varepsilon \rightarrow 0^{-}$.

The $G^{\prime \prime}$ minimum had also been modeled by Mason and Weitz (1995), and additional discussions of $G^{\prime}$ and $G^{\prime \prime}$ in the vicinity of the $G^{\prime \prime}$ minimum are given in Crassous et al. (2008a).

\section{Discussion}

The strength of the proposed powerlaw spectrum is in its abilities to rationalize linear viscoelastic data in the approach of the glass $(\varepsilon<0)$ and to make detailed predictions. Near-glass viscoelasticity follows the proposed powerlaw function, and it does not seem to be necessary there to account for a very slow relaxation mode, which typically governs the glass state $(0 \leq \varepsilon)$.

The data presented here are dominated by the $\alpha$-relaxation behavior and its strong $\varepsilon$ dependence. The $\alpha$ modes can be superimposed by applying an $\varepsilon$-dependent time shift. However, the entire spectrum cannot be shifted because of the widening gap between $\alpha$ and $\beta$ relaxation.

The BSW spectrum has been known for some time, but its occurrence was thought to be a specific property of monodisperse polymers with long linear flexible chains both, in the melt state or in solution (Baumgärtel et al. 1990, 1992; Abdel-Goad et al. 2004; Friedrich et al. 2008). The physical image of such a polymer is that its individual long-chain molecules are caged in a tubelike space. Relaxation of stress in the linear polymer is explained by the gradual escape of chains from their cage. Several different escape mechanisms are envisioned to contribute to this process (Doi 1981; Milner and McLeish 1998; Likhtman and McLeish 2002). This study shows that the relaxation properties of the nearglass and the monodisperse linear polymer are similar. Both material groups can be, to a close approximation, represented by the same BSW spectrum but different parameter space. The similarity in the relaxation patterns is, to our current understanding, purely coinci- dental since the physical origin of the BSW parameters differ for the two groups of materials. The longest relaxation time diverges for linear polymers with molecular weight, $\tau_{\max } \sim M^{3.4}$ (Doi 1981), and for near-glass colloidal suspensions with the inverse distance from the glass, $\tau_{\max } \sim(-\varepsilon)^{-\gamma}$. MCT predicts exponent values in the order of $\gamma=2.4$.

The study of density correlations of Franosch and Götze (1999) already suggests the powerlaw structure of the relaxation time spectrum. The linear moduli are the Fourier transforms of the shear stress autocorrelation function, which, in MCT, is quadratic in the density correlators. The same exponents appear in stress and density correlators as can be checked by Taylor expansion of the correlators around their respective glass plateaus.

The specific rheological features of the near-glass become most evident when comparing relaxation phenomena of the glass transition and with those of gelation. The longest relaxation time, $\tau_{\max }$, and zero shear viscosity, $\eta_{0}$, diverge for both transitions, the nearglass $\tau_{\max } \sim \eta_{0} \sim(-\varepsilon)^{-\gamma}$ (MCT) and the approach of the gel point from the liquid side, $\tau_{\max } \sim \eta_{0}^{1 /\left(1-n_{\mathrm{cg}}\right)} \sim$ $(-\varepsilon)^{-s /\left(1-n_{\mathrm{cg}}\right)}$, where $s$ is the critical exponent of the diverging viscosity (Winter 1987). The characteristic $\alpha$-relaxation $G^{\prime \prime}$ peak shifts to lower and lower frequencies while maintaining its height. The longest relaxation time correlates with the position of the $G^{\prime \prime}$ peak, $\tau_{\alpha} \cong 1 / n_{\alpha} \omega_{\alpha, \max }$, as shown in Eq. 15 . There is no counterpart for the diverging $G^{\prime \prime}$ peak in gelation. Gelation is characterized by the stretching of the relaxation time spectrum into a powerlaw at the gel point, which extends to infinite time (critical gel relaxation modulus $G(t)=S t^{-n_{\mathrm{cg}}}$; stiffness $S$ and exponent value $0<$ $n_{\mathrm{cg}}<1$ ) (Chambon and Winter 1985, 1987; Winter and Chambon $1986 \mathrm{a}, \mathrm{b})$. The specific relaxation patterns differ distinctly for the two phenomena.

\section{Conclusions}

The empirical rheological constitutive equation, Eq. 5, differs significantly from previous models of near-glass dynamics, but it does not require any new parameters. Parameters and their respective values are identical with the known parameters of MCT. No new parameter was added and none was removed. The new finding as reported here concerns the interrelation of these parameters for glass-forming materials in the approach of their glass state. The distinct features of the nearglass dynamics allow a clear differentiation between glass transition and gelation in soft matter. 
Acknowledgements HHW acknowledges NSF support (CBET0651888) and the 2007 Summer School at the Aspen Center for Physics. We also thank the Deutsche Forschungsgemeinschaft, Forschergruppe 608 "Nichtlineare Dynamik," Bayreuth, and IRTG 667 "Soft Condensed Matter Physics" at Konstanz, for financial support.

\section{References}

Abdel-Goad M, Pyckhout-Hintzen W, Kahle S, Allgaier J, Richter D, Fetters LJ (2004) Rheological properties of 1,4polyisoprene over a large molecular weight range. Macromolecules 37:8135-8144

Baumgärtel M, Winter HH (1989) Determination of discrete relaxation and retardation time spectra from dynamic mechanical data. Rheol Acta 28:511-519

Baumgärtel M, Schausberger A, Winter HH (1990) The relaxation of polymers with linear flexible chains of uniform length. Rheol Acta 29:400 408

Baumgärtel M, DeRosa ME, Machado J, Masse M, Winter HH (1992) The relaxation time spectrum of nearly monodisperse polybutadiene melts. Rheol Acta 31:75-82

Bengtzelius U, Götze W, Sjölander A (1984) Dynamics of supercooled liquids and the glass transition. J Phys C 17:5915

Boltzmann L (1874) Zur Theorie der elastischen Nachwirkungen. Sitzungsber kaiserlich Akad Wissen Math Naturwissen 70:275-306

Brambilla G, El Masri D, Pierno M, Berthier L, Cipelletti L, Petekidis G, Schofield AB (2009) Probing the equilibrium dynamics of colloidal hard spheres above the mode-coupling glass transition. Phys Rev Let 102:085703

Carri G, Winter HH (1997) Mapping of the relaxation patterns of polymer melts with linear flexible molecules of uniform length. Rheol Acta 36:330-344

Chambon F, Winter HH (1985) Stopping of crosslinking reaction in a PDMS polymer at the gel point. Polym Bull 13:499-503

Chambon F, Winter HH (1987) Linear viscoelasticity at the gel point of a crosslinking PDMS with imbalanced stoichiometry. J Rheol 31:683-697

Crassous JJ, Siebenbürger M, Ballauff M, Drechsler M, Hajnal D, Henrich O, Fuchs M (2006a) Thermosensitive core-shell particles as model systems for studying the flow behavior of concentrated colloidal dispersions. J Chem Phys 125:204906

Crassous JJ, Ballauff M, Drechsler M, Schmidt J, Talmon $Y$ (2006b) Imaging the volume transition in thermosensitive core-shell particles by cryo-transmission electron microscopy. Langmuir 22:2403

Crassous $J J$, Siebenbürger $M$, Ballauff $M$, Drechsler $M$, Hajnal D, Henrich O, Fuchs M (2008a) Shear stresses of colloidal dispersions at the glass transition in equilibrium and in flow. J Chem Phys 128:204902

Crassous $J \mathbf{J}$, Wittemann $A$, Siebenbürger $M$, Schrinner $M$, Drechsler $M$, Ballauff $M(2008 \mathrm{~b})$ Direct imaging of temperature-sensitive core-shell latexes by cryogenic transmission electron microscopy. Colloid Polym Sci 286:805

Doi M (1981) Explanation for the 3.4 power law of viscosity of polymeric liquids on the basis of the tube model. J Polym Sci Polym Lett Ed 19:265

Ferry JD (1980) Viscoelastic properties of polymers, 3rd edn. Wiley, New York
Franosch T, Götze W (1999) Relaxation rate distributions for supercooled liquids. J Phys Chem B 103:4011

Friedrich C, Waizenegger F, Winter HH (2008) Relaxation patterns of long, linear, flexible, monodisperse polymers: BSW spectrum revisited. Rheol Acta 47(8):909-916

Fuchs M, Cates ME (2002) Theory of nonlinear rheology and yielding of dense colloidal suspensions. Phys Rev Lett 89:248304

Fuchs M, Hofacker I, Latz A (1992) Primary relaxation in a hardsphere system. Phys Rev A 45:898

Götze W (1991) Liquids, freezing and glass transition. In: Hansen JP, Levesque D, Zinn-Justin J (eds) Session LI of Les Houches summer schools of theoretical physics. NorthHolland, Amsterdam, pp 287

Götze W, Sjögren L (1992) Relaxation processes in supercooled liquids. Rep Prog Phys 55:241

Koumakis N, Schofield AB, Petekidis $G$ (2008) Effects of shear induced crystallization on the rheology and ageing of hard sphere glasses. Soft Matter 4:2008-2018

Larson RG (1999) The structure and rheology of complex fluids. Oxford University Press, Oxford

Likhtman A, McLeish TCB (2002) Quantitative theory for linear dynamics of linear entangled polymers. Macromolecules 35:6332

Mason TG, Weitz DA (1995) Linear viscoelasticity of hard sphere suspensions near the glass transition. Phys Rev Lett 75:2770-2773

Mayer P, Miyazaki K, Reichman DR (2006) Cooperativity beyond caging: generalized mode coupling rheory. Phys Rev Lett 97:095702

Milner ST, McLeish TCB (1998) Reptation and contour-length fluctuations in melts of linear polymers. Phys Rev Lett $81: 725$

Schweizer KS (2007) Dynamical fluctuation effects in glassy colloidal suspensions. Curr Opin Colloid Interface Sci 12:297

Schweizer KS, Saltzman EJ (2003) Entropic barriers, activated hopping, and the glass transition in colloidal suspensions. J Chem Phys 119:1181-1196

Siebenbürger M, Fuchs M, Winter HH, Ballauff M (2009) Viscoelasticity and shear fow of concentrated, non-crystallizing colloidal suspensions: comparison with mode-coupling theory. J Rheol 53:707-726

van Megen W, Underwood SM (1994) Glass-transition in colloidal hard spheres-measurement and modecouplingtheory analysis of the coherent intermediate scattering function. Phys Rev E 49:4206-4220

van Megen W, Underwood SM, Pusey PN (1991) Non-ergodicity parameters of colloidal glasses. Phys Rev Lett 67:15861589

von Schweidler E (1907) Studien über die Anomalien im Verhalten der Dielektrika. Ann Phys (Leipzig) 24:711-770

Winter HH (1987) Evolution of rheology during chemical gelation. Prog Colloid Polym Sci 75:104-110

Winter HH, Chambon F (1986a) Analysis of linear viscoelasticity of a crosslinking polymer at the gel point. $J$ Rheology 30:367382

Winter HH, Chambon F (1986b) Rheology of crosslinking polymers at the gel point. Proc Bi-annual Meeting Polymer Networks Group, Elsinore

Winter HH, Mours M (1997) Rheology of polymers near their liquid-solid transitions. Adv Polym Sci 134:165-234

Winter HH, Mours M (2006) The cyber infrastructure initiative for rheology. Rheol Acta 45:331-338 